\title{
Acute myocardial infarction versus other cardiovascular events in community- acquired pneumonia
}

\author{
Stefano Aliberti ${ }^{1}$, Julio Ramirez ${ }^{2}$, Roberto Cosentini ${ }^{3}$, Vincenzo Valenti ${ }^{4}$, \\ Antonio Voza ${ }^{5}$, Paolo Rossi ${ }^{6}$, Daiana Stolz ${ }^{7}$, Delfino Legnani ${ }^{8}$, Alberto Pesci $^{1}$, \\ Luca Richeldi ${ }^{9,10}$, Paula Peyrani ${ }^{2}$, Fernando Maria Massari ${ }^{11}$ and \\ Francesco Blasi ${ }^{12}$
}

Affiliations: ${ }^{1}$ Health Science Department, University of Milan Bicocca, Respiratory Unit, AO San Gerardo, Monza, Italy. ${ }^{2}$ Division of Infectious Diseases, Department of Medicine, University of Louisville, Louisville, KY, USA. ${ }^{3}$ Emergency Medicine Unit, IRCCS Fondazione Ospedale Maggiore Policlinico Cà Granda, Milan, Italy. ${ }^{4}$ Pulmonary Unit, University of Milan, IRCCS Policlinico San Donato, San Donato Milanese, Milan, Italy. ${ }^{5}$ Emergency Department, IRCCS Humanitas Research Hospital, Rozzano, Italy. ${ }^{6}$ Internal Medicine Department, Azienda Ospedaliero-Universitaria “S. Maria della Misericordia", Udine, Italy. ${ }^{7}$ Clinic of Respiratory Medicine and Pulmonary Cell Research, University Hospital Basel, Basel, Switzerland. ${ }^{8}$ Department of Biomedical and Clinical Sciences, University of Milan, Luigi Sacco Hospital, Milan, Italy. ${ }^{9}$ National Institute for Health Research Respiratory Biomedical Research Unit, Southampton, UK. ${ }^{10}$ Centre for Rare Lung Disease, University of Modena and Reggio Emilia, AO Policlinico, Modena, Italy. ${ }^{11}$ UOC Malattie Cardiovascolari, Università degli Studi di Milano, IRCCS Fondazione Ospedale Maggiore Policlinico Cà Granda, Milan, Italy. ${ }^{12}$ Department of Pathophysiology and Transplantation, Università degli Studi di Milano, IRCCS Fondazione Ospedale Maggiore Policlinico Cà Granda, Milan, Italy.

Correspondence: Stefano Aliberti, Health Science Department, University of Milan Bicocca, Respiratory Unit, A0 San Gerardo, Via Pergolesi 33, 20052, Monza, Italy. E-mail: stefano.alibertiQunimib.it

ABSTRACT The aim of the present study was to define the prevalence, characteristics, risk factors and impact on clinical outcomes of acute myocardial infarction (AMI) versus other cardiovascular events (CVEs) in patients with community-acquired pneumonia (CAP).

This was an international, multicentre, observational, prospective study of CAP patients hospitalised in eight hospitals in Italy and Switzerland. Three groups were identified: those without CVEs, those with AMI and those with other CVEs.

Among 905 patients, 21 (2.3\%) patients experienced at least one AMI, while 107 (11.7\%) patients experienced at least one other CVE. Patients with CAP and either AMI or other CVEs showed a higher severity of the disease than patients with CAP alone. Female sex, liver disease and the presence of severe sepsis were independent predictors for the occurrence of AMI, while female sex, age >65 years, neurological disease and the presence of pleural effusion predicted other CVEs. In-hospital mortality was significantly higher among those who experienced AMI in comparison to those experiencing other CVEs ( $43 \%$ versus $21 \%, \mathrm{p}=0.039$ ). The presence of AMI showed an adjusted odds ratio for in-hospital mortality of $3.57(\mathrm{p}=0.012)$ and for other CVEs of $2.63(\mathrm{p}=0.002)$.

These findings on AMI versus other CVEs as complications of CAP may be important when planning interventional studies on cardioprotective medications.

@ERSpublications

Acute myocardial infarction is associated with specific risk factors and accounts for worse outcomes in CAP patients http://ow.ly/QhT2t

This article has supplementary material available from openres.ersjournals.com

Received: April 302015 | Accepted after revision: July 212015

Support statement: Daiana Stolz was supported by the Swiss National Foundation (grant PP00P3_128412/1).

Conflict of interest: Disclosures can be found alongside this article at openres.ersjournals.com

Copyright $\odot$ ERS 2015. This article is open access and distributed under the terms of the Creative Commons Attribution Non-Commercial Licence 4.0. 


\section{Introduction}

While mortality for community-acquired pneumonia (CAP) decreased sharply after the introduction of antibiotics in the 1940s, the overall mortality has either remained stable or increased since 1950 [1]. Thus, there is a clear need to explore new approaches and test strategies to reduce mortality by reducing complications related to pneumonia.

The current literature indicates that cardiovascular events (CVEs) may happen in patients with pneumonia both during hospitalisation and after discharge, with an absolute rate of events varying broadly from $10 \%$ to $30 \%$ across different cohorts [2]. CVEs have a strong impact on patients' outcomes, leading to an increase in both short-term and 30-day mortality [3]. Notably, heterogeneity has been reported in literature concerning the types of CVE occurring in CAP patients, which include acute myocardial infarction (AMI), acute heart failure and cardiac arrhythmias.

Special attention from both a clinical and a research point of view has been given to the occurrence of AMI in patients with pneumonia [2]. Plaque rupture, in situ thrombus formation and consequent myocardial ischaemia may be triggered or worsened during an episode of pneumonia and, thus, lead to the occurrence of AMI. However, acute or persistent inflammation and hypoxaemia during an episode of CAP could lead to diffuse organ abnormalities with cellular dysfunction and, thus, the occurrence of other CVEs, such as cardiac arrhythmias. A head-to-head comparison of AMI versus other CVEs in terms of both specific risk factors for their occurrence and patient presentation and outcomes has not been previously conducted, although it would be crucial to design accurate interventional studies on cardioprotective medications.

We designed a study with the objective of defining the prevalence, characteristics, risk factors and impact on clinical outcomes of AMI versus other CVEs in hospitalised patients with CAP.

\section{Materials and methods}

\section{Study design and data collection}

This was an international, multicentre, observational, prospective cohort study of consecutive patients hospitalised with a diagnosis of CAP in eight acute care hospitals in Italy and Switzerland from October 2009 to October 2012 (online supplementary material). Patients $\geqslant 18$ years of age and satisfying the criteria for CAP were included in the study. Patients with hospital-acquired pneumonia, defined as pneumonia that developed $48 \mathrm{~h}$ after the current hospitalisation or pneumonia that developed in a patient who was discharged from the hospital within the prior 14 days of the current hospitalisation, were excluded from the study. Patients with a diagnosis of healthcare-associated pneumonia were included in the study [4]. The institutional review boards of all the hospitals approved the study and patients signed an informed consent.

The clinical management of patients, laboratory evaluations and antimicrobial therapy were performed according to the current standard of care for patients with CAP [5]. Patients were seen daily during their hospital stay by one or more of the investigators who recorded clinical data (online supplementary material). A telephone call or a visit to the clinic was performed by one of the investigators in each study centre 30 days after the diagnosis of CAP was made in order to determine the possible development of adverse events after discharge and either rehospitalisation or death. Study definitions are reported in the online supplementary material, including those of acute respiratory failure (ARF) and severe sepsis.

\section{Microbiological analysis and empiric antibiotic therapy}

Microbiological examinations were performed on sputum, urine and blood during the first $24 \mathrm{~h}$ after admission and according to standards of practice. Identification of microorganisms and susceptibility testing were performed according to standard methods (online supplementary material) [6]. Empiric antibiotic therapy was administered as soon as the diagnosis of pneumonia was reached in the emergency department. The empiric antibiotic treatment was evaluated for compliance with the European Respiratory Society guidelines [5].

\section{CVEs and study groups}

CVEs were considered if any of the following was present either on hospital admission or during hospitalisation: 1) AMI; 2) acute cardiogenic pulmonary oedema; 3) new arrhythmia; 4) acute worsening of a long-term arrhythmia; 5) cerebrovascular accident; or 6) pulmonary embolism. Criteria for AMI were defined as detection of rise and/or fall of troponin with at least one value above the 99th percentile of the upper reference limit together with evidence of myocardial ischaemia with at least one of the following: 1) symptoms of ischaemia; 2) ECG changes indicative of new ischaemia (new ST-T changes or new left bundle branch block); 3) development of pathological Q-waves in the ECG; or 4) imaging evidence of new 
loss of viable myocardium or new regional wall motion abnormality [7]. The process to define other CVEs is reported in the online supplementary material.

Three groups of patients were identified according to the presence and type of CVE either on admission or during hospitalisation: patients without CVE ("no CVE" group); patients with CVE excluding AMI ("other CVE" group); and patients with at least an AMI ("AMI" group). Patients experiencing a CVE triggered by AMI were included in the AMI group.

\section{Study outcomes}

The following were considered as the study outcomes.

\section{Time to clinical stability}

A patient was considered to reach clinical stability when the following criteria were met in a single day during hospitalisation: 1) improved clinical signs (cough and shortness of breath); 2) the patient was afebrile for $\geqslant 8 \mathrm{~h} ; 3$ ) improving leukocytosis (decreased $\geqslant 10 \%$ from the previous day), C-reactive protein or procalcitonin; and 4) tolerating oral intake [8]. Criteria for clinical stability were evaluated daily during the first 7 days of hospitalisation.

\section{Length of hospital stay}

Length of hospital stay was considered as the number of days from the date of admission to the date of discharge.

\section{In-hospital mortality}

In-hospital mortality was considered if death by any cause occurred during hospitalisation. Patients were followed from the day of admission to day 30; those who remained hospitalised for $>30$ days were considered alive.

\section{Clinical failure}

The definition of clinical failure is reported in the online supplementary material.

\section{Statistical analysis}

All data were statistically analysed using SPSS (version 18.0) for Mac (IBM, Armonk, NY, USA). Descriptive statistics were reported at baseline with continuous data expressed as mean \pm SD for normally distributed data, median (interquartile range (IQR)) for skewed data and categorical data expressed as counts. Patient characteristics were compared between groups. Differences of continuous data between groups were evaluated by unpaired t-tests or Mann-Whitney U-tests (two groups), and ANOVA or Kruskal-Wallis tests (three groups). Differences of categorical variables between two or more groups were analysed using the Chi-squared test or Fisher's exact test where appropriate. Potential predictors of the occurrence of CVEs and mortality were investigated with a multivariable binomial logistic regression analysis. A p-value $<0.05$ was considered statistically significant.

\section{TABLE 1 Prevalence of hospitalised patients with community-acquired pneumonia experiencing cardiovascular events either on admission or during hospitalisation}

\begin{tabular}{|c|c|c|c|}
\hline & On admission & During hospitalisation & Total $^{\#}$ \\
\hline Acute myocardial infarction & 12 (1.3) & $9(1)$ & $21(2.3)$ \\
\hline Acute cardiogenic pulmonary oedema & $18(2)$ & 15 (1.7) & 33 (3.7) \\
\hline Arrhythmia & $45(5)$ & $31(3.4)$ & $77(8.6)$ \\
\hline New arrhythmia & $41(4.5)^{\pi}$ & $31(3.4)^{\S}$ & $72(8)$ \\
\hline Long-term arrhythmia & $5(0.6)^{+}$ & $2(0.2)^{f}$ & $7(0.8)$ \\
\hline Pulmonary embolism & $4(0.4)$ & $8(0.9)$ & 12 (1.3) \\
\hline Cerebrovascular accident & $6(0.7)$ & $3(0.2)$ & $9(1.1)$ \\
\hline \multicolumn{4}{|c|}{$\begin{array}{l}\text { Data are presented as } \mathrm{n}(\%) \text { patients. Three patients experienced a new arrhythmia both on admission and } \\
\text { during hospitalisation. }{ }^{*} \text { : either on admission or during hospitalisation; }{ }^{\uparrow} \text { : nine flutters and } 32 \text { atrial } \\
\text { fibrillations; }{ }^{+} \text {: three flutters and two atrial fibrillation; }{ }^{\S} \text { : three chaotic atrial rhythms, one paroxysmal } \\
\text { supraventricular tachycardia, two ventricular tachycardias and } 25 \text { atrial fibrillation; } f: \text { two atrial } \\
\text { fibrillations. }\end{array}$} \\
\hline
\end{tabular}




\section{Results}

Prevalence of CVEs

Among the 905 patients enrolled during the study period, 128 (14\%) experienced at least one CVE, either on admission (8.7\%) or during hospitalisation (6.4\%) (table 1). A total of 21 (2.3\%) patients experienced at least one AMI, while 107 (11.7\%) patients at least one other CVE (online supplementary figure A). Three patients experienced an acute cardiogenic pulmonary oedema and seven an arrhythmia triggered by AMI and, thus, were categorised in the AMI group. Median (IQR) time to the development of AMI during hospitalisation was 4 (1-8.5) days and for other CVEs was 4 (3-8) days after the diagnosis of pneumonia. Among patients who experienced AMI, 11 (52\%) had a ST segment elevation myocardial infarction (STEMI). Among those, aspirin was given in 10 (91\%) patients, low weight molecular heparin (LWMH) in eight (73\%) and $\beta$-blockers in seven (64\%), while seven (64\%) underwent percutaneous coronary intervention (PCI). Among 10 patients with non-ST segment elevation myocardial infarction (NSTEMI), aspirin was given in seven (70\%), nitrates in six (60\%), $\beta$-blockers in five (50\%), clopidogrel in five $(50 \%)$ and LWMH in four $(40 \%)$, while one $(10 \%)$ patient underwent PCI.

\section{Patient characteristics}

Baseline demographics; comorbidities; disease severity; clinical, laboratory and radiological findings on admission; microbiology; and antibiotic therapy of the study groups are summarised in online supplementary tables A-D. In comparison to patients with other CVEs, those with AMI: showed a significantly higher severity of the disease on admission, according to both the Pneumonia Severity Index (PSI) and the presence of severe sepsis; had a higher prevalence of chronic renal failure $(\mathrm{p}=0.015)$, liver disease $(\mathrm{p}<0.001)$ and thrombocytosis $(\mathrm{p}=0.006)$; and had significantly higher values of lactate dehydrogenase $(p=0.036)$, triglycerides $(p=0.041)$, cholesterol $(p=0.034)$ and glucose $(p=0.033)$ on admission (table 2).

A significant increasing trend in the proportion of patients with AMI and other CVEs was found across PSI risk classes and CURB-65 score (figure 1). A total of 3.8\% and 5.7\% of patients with CURB-65 scores 0-1 and PSI risk classes I-III, respectively, developed at least one CVE either on admission or during hospitalisation. No AMI was identified among patients in PSI risk classes I-III, while two patients with CURB-65 scores 0-1 had AMI. A significant increasing trend in the proportion of patients with AMI and other CVEs was found moving from patients with neither ARF nor severe sepsis, to those with only ARF, and those with both ARF and severe sepsis (figure 2).

\section{Risk factors for AMI versus other CVEs}

Findings significantly associated with the occurrence of AMI and other CVEs on univariate analysis are reported in online supplementary table E. Independent predictors of the occurrence of AMI and other CVE are show in table 3, after adjustment for several confounders. Female sex (adjusted odds ratio (aOR) 2.72, $\mathrm{p}=0.044)$, liver disease ( $\mathrm{aOR} 5.82, \mathrm{p}=0.003$ ) and the presence of severe sepsis $(\mathrm{aOR} 4.33, \mathrm{p}=0.005)$ on admission were independent predictors for the occurrence of AMI, while female sex ( $a \mathrm{OR} 1.77, \mathrm{p}=0.009$ ), age $>65$ years $(\mathrm{aOR} 2.88, \mathrm{p}=0.005)$, neurological disease $(\mathrm{aOR} 1.63, \mathrm{p}=0.047)$ and the presence of pleural effusion (aOR 1.64, $\mathrm{p}=0.026$ ) on admission were independent predictors for the occurrence of other CVE.

\section{Impact on clinical outcomes}

Median (IQR) time to clinical stability was longer in patients experiencing AMI (8 (5-8) days) in comparison to those experiencing other CVEs (6 (3-8) days) $(\mathrm{p}=0.050)$. Length of hospital stay was 10 (8-17) days in patients experiencing AMI and 11 (8-16) days in patients experiencing other CVEs $(\mathrm{p}=0.751)$. A total of $81(9 \%)$ patients died during hospitalisation in the entire study population. In-hospital mortality was significantly higher among those who experienced AMI in comparison to those experiencing other CVE ( $43 \%$ versus $21 \%, \mathrm{p}=0.039$ ), along with clinical failure (figure 3 ). Among patients with AMI who died, four had a STEMI and five a NSTEMI. Univariate analysis for in-hospital mortality is shown in online supplementary table F. On logistic regression analysis, after adjustment for severity of the disease, immunocompromised state and risk factors for multidrug-resistant pathogens, the presence of AMI either on admission or during hospitalisation showed an aOR for in-hospital mortality of 3.57 ( $\mathrm{p}=0.012)$ and for other CVEs of $2.63(\mathrm{p}=0.002)$ (figure 4).

\section{Discussion}

This study indicates that AMI is less prevalent (2.3\%) in CAP patients than other CVEs (11.7\%) but is associated with a significantly higher severity of the disease on admission and accounts for a significantly higher in-hospital mortality (43\%). The occurrence of AMI in CAP patients increases almost four-fold the risk of death during hospitalisation, while other CVEs increase it 2.6-fold. Specific and independent risk factors for the occurrence of AMI are severe sepsis and previous history of liver disease, while those for 


\begin{tabular}{|c|c|c|c|}
\hline & AMI & Other CVE & p-value \\
\hline Patients n & 21 & 107 & \\
\hline \multicolumn{4}{|l|}{ Demographics } \\
\hline Females & 12 (57) & $55(51)$ & 0.630 \\
\hline Age years & $79(72-85)$ & $83(75-88)$ & 0.258 \\
\hline \multicolumn{4}{|l|}{ Severity on admission } \\
\hline PSI risk class $\mathrm{V}$ & $17(81)$ & $53(50)$ & 0.007 \\
\hline Severe sepsis & $15(71)$ & $43(40)$ & 0.009 \\
\hline \multicolumn{4}{|l|}{ Comorbidities } \\
\hline Active neoplastic disease & $5(24)$ & $14(13)$ & 0.206 \\
\hline Chronic obstructive pulmonary disease & $9(43)$ & 35 (33) & 0.371 \\
\hline Diabetes mellitus & $4(19)$ & $25(23)$ & 0.666 \\
\hline Prior cerebrovascular accident & $10(48)$ & $33(31)$ & 0.137 \\
\hline Liver disease & $5(24)$ & $1(0.9)$ & $<0.001$ \\
\hline Neurological diseases & 7 (33) & $33(31)$ & 0.822 \\
\hline Renal disease & $2(9.5)$ & $10(9.3)$ & 0.980 \\
\hline Chronic renal failure & 7 (33) & $13(12)$ & 0.015 \\
\hline Immunosuppression ${ }^{\#}$ & $6(29)$ & $14(13)$ & 0.074 \\
\hline Family history of coronary artery disease & 6 (29) & $18(17)$ & 0.207 \\
\hline Essential arterial hypertension & $11(52)$ & $63(59)$ & 0.581 \\
\hline Congestive heart failure & $9(43)$ & $33(31)$ & 0.284 \\
\hline Coronary artery disease & $8(38)$ & $29(27)$ & 0.310 \\
\hline Prior acute myocardial infarction & $4(19)$ & $19(18)$ & 0.888 \\
\hline Atrial fibrillation & 6 (29) & $27(25)$ & 0.749 \\
\hline Hyperlipidaemia & 7 (33) & $19(18)$ & 0.105 \\
\hline \multicolumn{4}{|l|}{ Medications before admission } \\
\hline Aspirin & $10(48)$ & $41(38)$ & 0.426 \\
\hline$\beta$-blockers & 6 (29) & $26(24)$ & 0.679 \\
\hline ACE inhibitors & $6(29)$ & $36(34)$ & 0.651 \\
\hline Warfarin & 2 (9.5) & $5(4.7)$ & 0.371 \\
\hline Heparin & $0(0)$ & $5(4.7)$ & 0.312 \\
\hline Antiplatelets & $1(4.8)$ & $15(14)$ & 0.241 \\
\hline Statins & $4(19)$ & $10(10)$ & 0.253 \\
\hline \multicolumn{4}{|l|}{ Clinical and laboratory data on admission } \\
\hline Platelets $>450000$ per $\mu \mathrm{L}$ & $4(20)$ & $4(3.8)$ & 0.006 \\
\hline Lactate dehydrogenase $\mathrm{mg} \cdot \mathrm{dL}^{-1}$ & 510 (389-725) & $391(321-502)$ & 0.036 \\
\hline Triglycerides $\mathrm{mg} \cdot \mathrm{dL}^{-1}$ & 108 (93-157) & $94(72-117)$ & 0.041 \\
\hline Glucose $\mathrm{mg} \cdot \mathrm{dL}^{-1}$ & $168(132-225)$ & $128(109-183)$ & 0.033 \\
\hline Cholesterol $\mathrm{mg} \cdot \mathrm{dL}^{-1}$ mean $\pm \mathrm{SD}$ & $178 \pm 72$ & $148 \pm 35$ & 0.034 \\
\hline
\end{tabular}

Data are presented as $\mathrm{n}(\%)$ or median (interquartile range), unless otherwise stated. AMI: acute myocardial infarction; PSI: Pneumonia Severity Index; ACE: angiotensin-converting enzyme. " : presence of at least one of active cancer, asplenia or HIV infection.

other CVEs are advance age, the presence of pleural effusion and a previous history of neurological disease. Finally, female sex is an independent risk factor for both AMI and other CVE.

The prevalence of AMI in our study confirmed data recently reported in a meta-analysis showing either AMI or unstable angina in $5 \%$ of patients with pneumonia [9]. Similarly, the $11.7 \%$ prevalence of other CVEs in our cohort fits with data from recent observational studies showing the occurrence of new or worsening of pre-existing arrhythmia in $1-11 \%$ and heart failure in 3-18\% [4, 9-12]. Our data support previous findings showing an increased short-term mortality in CAP patients with CVE and the need of promptly diagnose and treat them [9-13]. From a research point of view, our data highlighting significant differences in outcomes between patients with AMI versus other CVEs could be useful for planning future interventional studies. Aspirin, for instance, along with statins, heparin and $\beta$-blockers, could be part of a specific prophylactic scheme for CAP patients at risk for developing AMI, while antiarrhythmic drugs including amiodarone, calcium channel and $\beta$-blockers could be used for those at risk for arrhythmias.

Although atherosclerotic plaque instability could be one of the main factors in the development of AMI in the post-pneumonia period, the actual mechanisms by which pneumonia triggers myocardial ischaemia 


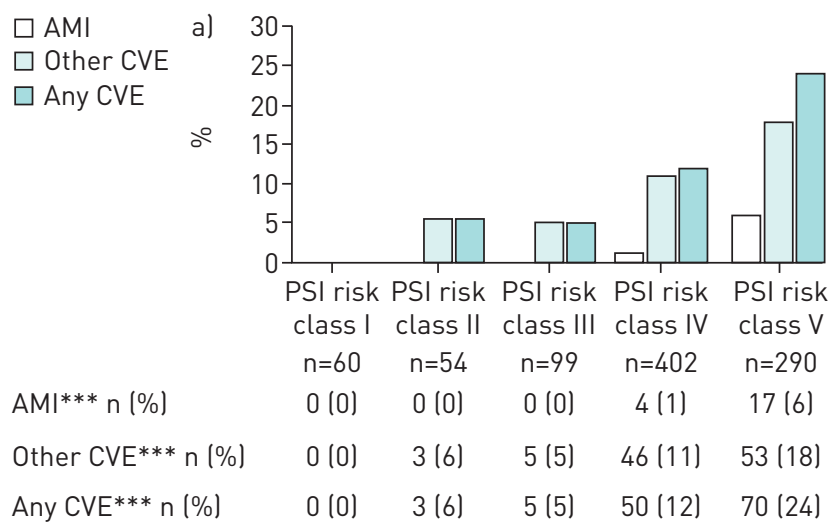

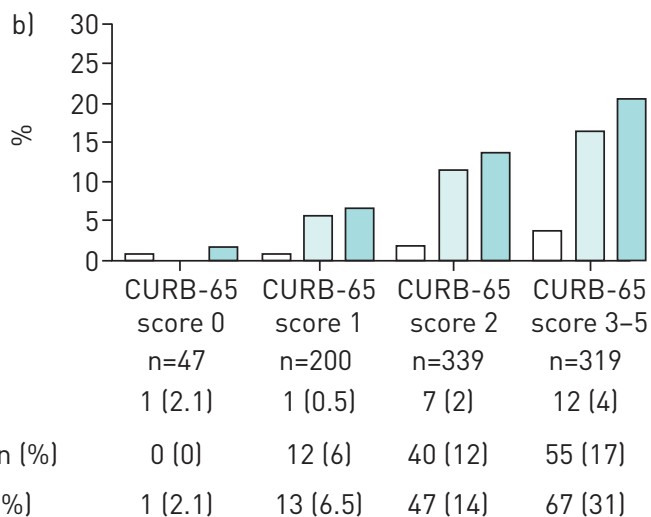

FIGURE 1 Prevalence of acute myocardial infarction (AMI) or other cardiovascular events (CVEs) according to a) Pneumonia Severity Index (PSI) risk classes and b) CURB-65 Score. ${ }^{* * *}$ : $p<0.001$ across PSI risk classes; ${ }^{*}: p=0.014$ across CURB-65 scores; : $p=0.001$ across CURB-65 score; ${ }^{+}: \mathrm{p}<0.001$ across CURB-65 scores.

have not yet been fully evaluated. Several speculations have been reported in the literature in recent years, focussing on the role of plaque disruption, demand ischaemia or endothelial dysfunction leading to vasoconstriction $[2,14,15]$. There may well be a fair amount of overlap between the mechanisms causing the two different types of CVEs and some pathophysiological aspects, including the role of the cardiac autonomic control and the pro-coagulation state, should be better evaluated in future translational studies.

If we were to delineate the two main characteristics of the typical patient with CAP to monitor for the onset of CVEs and to be enrolled in an interventional trial of cardioprotective drugs according to our results, these would be advanced age and severe pneumonia, especially in the presence of severe sepsis. Our data showed that one out of four patients in PSI risk class V had a CVE and that up to 6\% had an AMI. From a research point of view, the idea for future trials would be to focus attention on severe patients, such as those with PSI risk class V or CURB-65 score 3-5. From a clinical point of view, various reports have highlighted a clear difficulty in implementing both PSI and CURB-65 in daily practice, and a simple stratification of severity of CAP patients according to the presence of ARF and severe sepsis has instead been suggested by some authors and supported by recent data [16-20]. In the present study, we showed a prevalence of CVEs of $26 \%$ among CAP patients with both ARF and severe sepsis, and $7 \%$ prevalence of AMI.

We confirmed previous reports showing that coronary artery disease, pleural effusion, severe sepsis, age and female sex are independently associated with CVEs [2]. Female sex also seems to be an independent predictor of the occurrence of both AMI and other CVEs, and this is a completely new finding. Previous studies have showed that females with both ventilator-associated and community-acquired pneumonia are more likely to die than males [21, 22]. Recent data from the Community-Acquired Pneumonia Organization cohort study showed that hospitalised females with CAP take longer to improve clinically than male patients and experience higher 28-day mortality [22]. The higher occurrence of CVEs in

FIGURE 2 Prevalence of acute myocardial infarction (AMI) or other cardiovascular events (CVEs) according to the presence of acute respiratory failure (ARF) and severe sepsis (SS) on hospital admission. ***: $\quad p<0.001$ across the three groups.

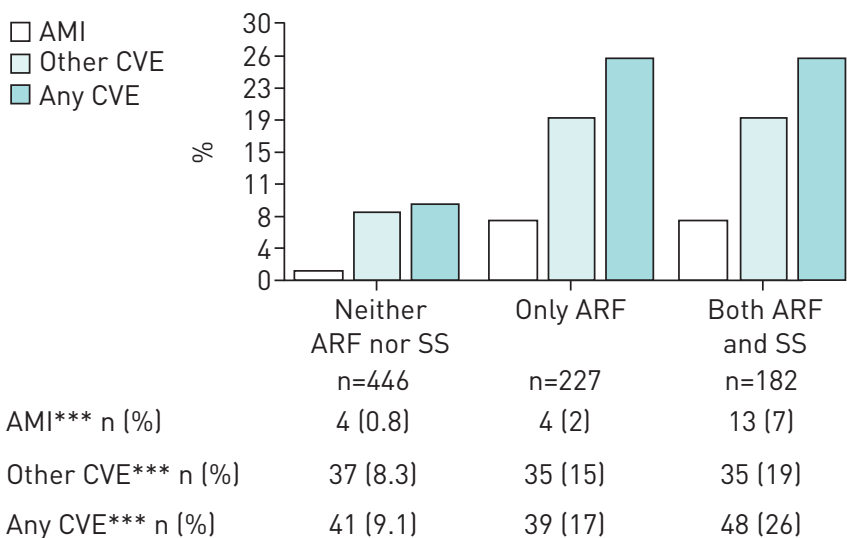


TABLE 3 Independent predictors for the occurrence of cardiovascular events (CVEs) either on admission or during hospitalisation on multivariable analysis

\begin{tabular}{|c|c|c|c|c|c|c|}
\hline & \multicolumn{2}{|c|}{$\mathrm{AMI}^{\#}$} & \multicolumn{2}{|c|}{ Other CVE } & \multicolumn{2}{|c|}{ Any $\mathrm{CVE}^{+}$} \\
\hline & aOR $(95 \% \mathrm{CI})$ & p-value & aOR $(95 \% \mathrm{CI})$ & p-value & aOR $(95 \% \mathrm{CI})$ & p-value \\
\hline Female sex & $2.72(1.02-7.25)$ & 0.044 & $1.77(1.15-2.70)$ & 0.009 & $1.85(1.24-2.77)$ & 0.003 \\
\hline Severe sepsis & $4.33(1.55-12.1)$ & 0.005 & & & $1.74(1.15-2.63)$ & 0.009 \\
\hline Pleural effusion & & & $1.64(1.01-2.53)$ & 0.026 & $1.63(1.08-2.45)$ & 0.020 \\
\hline Coronary artery disease & & & & & $1.74(1.08-2.81)$ & 0.023 \\
\hline
\end{tabular}

Factors included in the multivariable logistic regression analysis were age, sex, history of congestive heart failure, cerebrovascular disease, coronary artery disease, and severe sepsis and acute respiratory failure on admission. Nursing home residency was added for both any CVE and acute myocardial infarction (AMI). Liver disease and chronic renal failure were added only for AMI. Pleural effusion, history of neurological disease and atrial fibrillation were added for any CVE and other CVE. aOR: adjusted odds ratio. ${ }^{\#}$ : Nagelkerke $r^{2}=0.270 ;{ }^{\text {१: }}$ Nagelkerke $r^{2}=0.118$; ${ }^{+}$: Nagelkerke $r^{2}=0.147$.

females than males that we found in our cohort of CAP patients could be one explanation of these previous findings.

Our study has some limitations. Its design suffers of the missing of a complete evaluation of other risk factors for CVEs (including the analysis of the cardiac autonomic control and the pro-coagulation state) and long-term outcomes, such as the occurrence of CVEs and mortality at 1-year follow-up. Coronary angiography was not required to make a diagnosis of AMI and the universal definition of AMI has been chosen in our study according to both clinical practice and published literature [23]. In view of the sample size, the lack of the use of molecular microbiology (such as PCR of throat swabs/nasopharyngeal aspirates) and the low sensitivity/specificity of the adopted diagnostic techniques, we were not able to identify a correlation between CVEs and pathogens causing pneumonia, including respiratory viruses. Finally, due to the low number of bacteraemic patients in our study, we were not able to investigate the probable role of bacterial invasion of myocardium/plaque as recently hypothesised [24].

Although the impact of CVEs is a well-known problem in CAP patients, most of the literature is based on retrospective observational or administrative reports. This is the first multicentre, prospective, observational study that used standardised definitions of CVE, including AMI, that have also been reviewed by a committee of experts, including a cardiologist. Furthermore, several early and medium-term clinical outcomes have been evaluated, showing concordance among them in terms of results.

In conclusion, AMI seems to be associated with specific risk factors that are different from those associated with other CVE and, although less prevalent, accounts for significantly worse outcomes in hospitalised patients with CAP. A different approach for AMI versus other CVEs could be suggested, especially from a research point of view, to plan interventional studies on cardioprotective medications.

FIGURE 3 Clinical outcomes of the study population according to the three study groups. CVE: cardiovascular event; AMI: acute myocardial infarction. *: $p<0.001$ across the three groups.
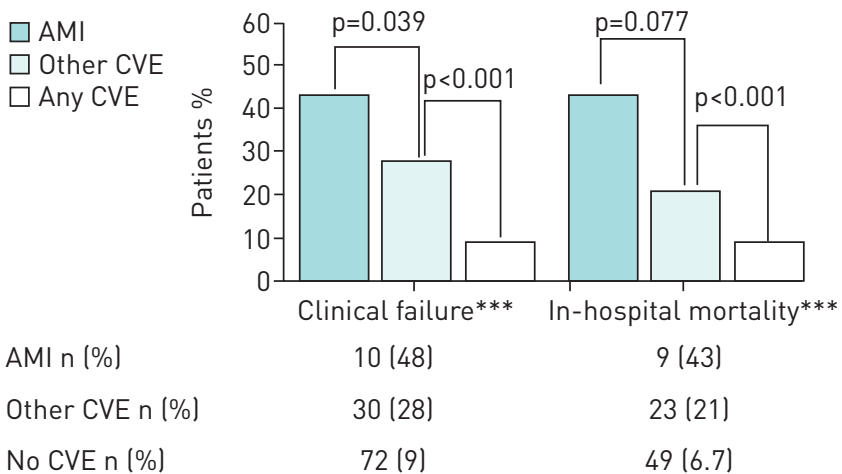


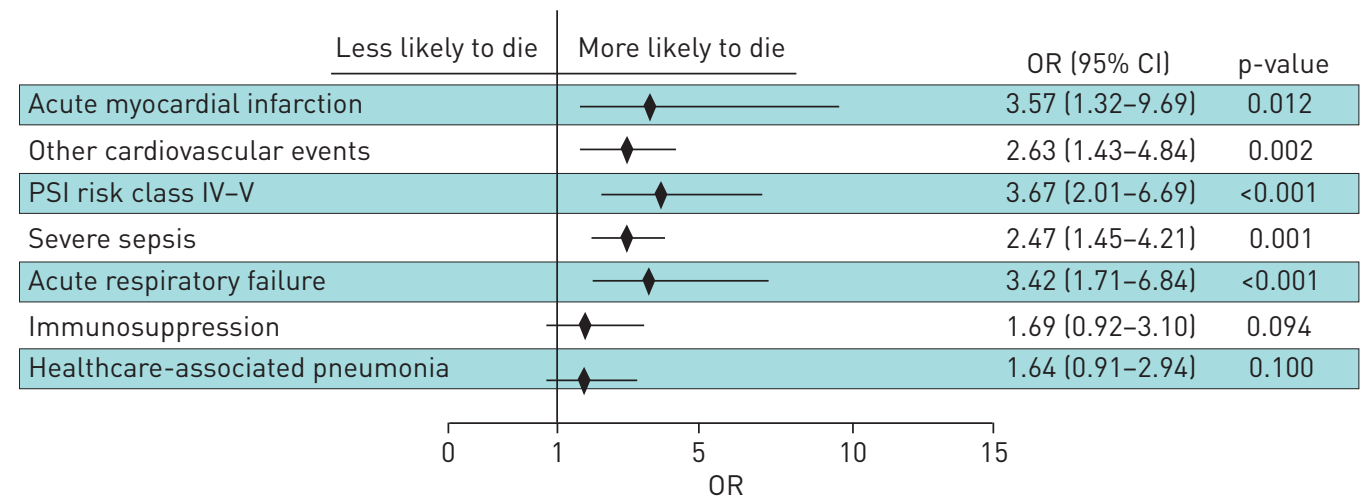

FIGURE 4 Multivariable logistic regression analysis for in-hospital mortality among the study population. Whiskers represented 95\% confidence intervals. PSI: Pneumonia Severity Index.

\section{Acknowledgments}

The authors acknowledge the assistance of Valeria Rossetti, Letizia Corinna Morlacchi and Fabio Giuliani (Department of Pathophysiology and Transplantation, Università degli Studi di Milano, IRCCS Fondazione Ospedale Maggiore Policlinico Cà Granda, Milan, Italy), Simona Perossi, Giulia Bonaiti, Giulia Suigo and Paola Faverio (Health Science Department, University of Milan Bicocca, Respiratory Unit, AO San Gerardo, Monza, Italy), Giulia Cervi and Riccardo Fantini (Centre for Rare Lung Disease, University of Modena and Reggio Emilia, AO Policlinico, Modena, Italy), Anna Maria Brambilla and Valter Monzani (Emergency Medicine Unit, IRCCS Fondazione Ospedale Maggiore Policlinico Cà Granda, Milan, Italy), Elena Magni (Department of Biomedical and Clinical Sciences, University of Milan, Luigi Sacco Hospital, Milan, Italy), and Elena Parazzini (Emergency Department, IRCCS Humanitas Research Hospital, Rozzano, Milan, Italy).

\section{References}

1 Armstrong GL, Conn LA, Pinner RW. Trends in infectious disease mortality in the United States during the 20th century. JAMA 1999; 281: 61-66.

2 Aliberti S, Ramirez JA. Cardiac diseases complicating community-acquired pneumonia. Curr Opin Infect Dis 2014; 27: 295-301.

3 Corrales-Medina VF, Musher DM, Wells GA, et al. Cardiac complications in patients with community-acquired pneumonia: incidence, timing, risk factors, and association with short-term mortality. Circulation 2012; 125: 773-781.

4 American Thoracic Society, Infectious Diseases Society of America. Guidelines for the management of adults with hospital-acquired, ventilatory-associated, and healthcare-associated pneumonia. Am J Respir Crit Care Med 2005; 171: $388-416$

5 Woodhead M, Blasi F, Ewig S, et al. Guidelines for the management of adult lower respiratory tract infections summary. Clin Microbiol Infect 2011; 17: Suppl. 6: 1-24.

6 National Committee for Clinical Laboratory Standards. Performance standards for antimicrobial susceptibility testing, 14th informational supplement. Approved standard M100-S14. Wayne, National Committee for Clinical Laboratory Standards, 2004.

7 Thygesen K, Alpert JS, White HD, et al. Universal definition of myocardial infarction. Circulation 2007; 116: 2634-2653.

8 Aliberti S, Zanaboni AM, Wiemken $\mathrm{T}$, et al. Criteria for clinical stability in hospitalised patients with community-acquired pneumonia. Eur Respir J 2013; 42: 742-749.

9 Corrales-Medina VF, Suh KN, Rose G, et al. Cardiac complications in patients with community-acquired pneumonia: a systematic review and meta-analysis of observational studies. PLoS Med 2011; 8: e1001048.

10 Musher DM, Rueda AM, Kaka AS, et al. The association between pneumococcal pneumonia and acute cardiac events. Clin Infect Dis 2007; 45: 158-165.

11 Griffin AT, Wiemken TL, Arnold FW. Risk factors for cardiovascular events in hospitalized patients with community-acquired pneumonia. Int J Infect Dis 2013; 17: e1125-e1129.

12 Viasus D, Garcia-Vidal C, Manresa F, et al. Risk stratification and prognosis of acute cardiac events in hospitalized adults with community-acquired pneumonia. J Infect 2013; 66: 27-33.

13 Ramirez J, Aliberti S, Mirsaeidi M, et al. Acute myocardial infarction in hospitalized patients with community-acquired pneumonia. Clin Infect Dis 2008; 47: 182-187.

14 Corrales-Medina VF, Musher DM, Shachkina S, et al. Acute pneumonia and the cardiovascular system. Lancet 2013; 381: 496-505.

15 Corrales-Medina VF, Madiid M, Musher DM. Role of acute infection in triggering acute coronary syndromes. Lancet Infect Dis 2010; 10: 83-92.

16 Lee RW, Lindstrom ST. A teaching hospital's experience applying the Pneumonia Severity Index and antibiotic guidelines in the management of community-acquired pneumonia. Respirology 2007; 12: 754-758.

17 Nadarajan P, Wilson L, Mohammed B, et al. Compliance in the measurement of CURB-65 in patients with community acquired pneumonia and potential implications for early discharge. Ir Med J 2008; 101: 144-146.

18 Barlow G, Nathwani D, Myers E, et al. Identifying barriers to the rapid administration of appropriate antibiotics in community-acquired pneumonia. J Antimicrob Chemother 2008; 61: 442-451.

19 Kolditz M, Ewig S, Höffken G. Management-based risk prediction in community-acquired pneumonia by scores and biomarkers. Eur Respir J 2013; 41: 974-984. 
20 Aliberti S, Brambilla AM, Chalmers JD, et al. Phenotyping community-acquired pneumonia according to the presence of acute respiratory failure and severe sepsis. Respir Res 2014; 15: 27.

21 Combes A, Luyt CE, Fagon JY, et al. Early predictors for infection recurrence and death in patients with ventilator-associated pneumonia. Crit Care Med 2007; 35: 146-154.

22 Arnold FW, Wiemken TL, Peyrani P, et al. Outcomes in females hospitalised with community-acquired pneumonia are worse than in males. Eur Respir J 2013; 41: 1135-1140.

23 McAllister DA, Maclay JD, Mills NL, et al. Diagnosis of myocardial infarction following hospitalisation for exacerbation of COPD. Eur Respir J 2012; 39: 1097-1103.

24 Brown AO, Mann B, Gao G, et al. Streptococcus pneumoniae translocates into the myocardium and forms unique microlesions that disrupt cardiac function. PLoS Pathog 2014; 10: e1004383. 